\title{
Estado de Salud Oral en Discapacitados Residentes en la Fundación Pequeño Cottolengo Don Orione, Paraguay
}

\author{
Oral Health Status of Disabled Residents at the \\ Fundación Pequeño Cottolengo Don Orione, Paraguay
}

\begin{abstract}
Nohelia María Pérez Bejarano*; Viviana Soledad Alarcón Gónzalez ${ }^{* * *}$; Marta Inés Ferreira Gaona*; Clarisse Virginia Díaz Reissner*; Pedro Duré"; Natalia Andriotti"; Dalia Moreno"; Luis Galeano**; Ariadnalis Mora*; Larissa Insfrán ${ }^{* *}$ \& Ruth Jara*
\end{abstract}

PÉREZ BEJARANO, N. M.; ALARCÓN GONZÁLEZ, V. S.; FERREIRA GAONA, M. I.; DÍAZ REISSNER, C. V.; DURÉ, P.; ANDRIOTTI, N.; MORENO, D.; GALEANO, L.; MORA, A.; INSFRÁN, L. \& JARA, R. Estado de salud oral en discapacitados residentes en la Fundación Pequeño Cottolengo Don Orione, Paraguay. Int. J. Odontostomat., 10(1):69-74, 2016.

RESUMEN: Viven con discapacidad aproximadamente 600 millones de individuos, cifra que va en aumento; de éstos el $80 \%$ reside en países de bajos ingresos. En el Paraguay, se estima que aproximadamente el $15 \%$ de la población presenta algún tipo de discapacidad. El objetivo fue describir el estado de salud oral de las personas con discapacidad que residen en la Fundación Pequeño Cottolengo Don Orione de Mariano Roque Alonso, Paraguay 2012. Se realizó un estudio observacional descriptivo de corte transversal en 37 niños, jóvenes y adultos en edades comprendidas entre 12 y 68 años. Fueron analizadas las lesiones de caried a través del índice CPO-D, lesiones no cariosas, lesiones de tejidos blandos y la presencia de factores de riesgo como nivel y frecuencia de higiene, placa microbiana, saliva y dieta. El índice CPO-D fue 16,24; se detectó presencia de lesiones no cariosas en $48,65 \%$ y fluorosis en 2,70 \%. Se encontraron fracturas a nivel de esmalte en $43,24 \%$ y la higiene oral fue deficiente medido por medio del índice de Loë y Silness. El $24,32 \%$ tenía saliva viscosa; el Índice de placa bacteriana fue de 2,04. Mientras que, en ninguno de los casos los momentos de azúcar superaron tres. El 100 \% de la población se cepilla una vez al día por la mañana antes del desayuno. En la población de estudio existen valores elevados en cuanto a caries y dientes perdidos, por tanto, se deben aumentar los programas de concientización en los cuidadores para promover la preservación de los dientes y la salud oral.

PALABRAS CLAVE: atención dental para personas con discapacidades, discapacidad intelectual, hogares de grupo.

\section{INTRODUCCIÓN}

En la sociedad, la discapacidad es considerada como un fenómeno social, debido a que éstas se deben integrar a la misma, implicando un conjunto de condiciones creadas por el ambiente social (Vázquez Barrios \& Cáceres, 2008), debido a la interacción entre las capacidades funcionales, entorno físico y social (Stang Alva, 2011).

Según la OMS viven con discapacidad 600 millones de individuos, cifra que va en aumento; de éstos el $80 \%$ reside en países de bajos ingresos (Asamblea Mundial de la Salud, 2005). En el Paraguay, se estima que aproximadamente el $15 \%$ de la población presenta algún tipo de discapacidad, pero existe un sub registro, pues menos del $1 \%$ figura en el Censo Nacional de Población y Viviendas 2002 (55 000 individuos), impidiendo de esta manera la visibilidad al Estado (Rivarola, 2012); dicha falencia está en conocimiento por la CEPAL, que señala que existió deficiencia en la fuente de datos (González, 2013). Cabe destacar, que el $50 \%$ de la discapacidad en nuestro país es causada por accidentes viales (Olmedo Zorilla, 2011). Finalmente, éstas personas resultan discriminadas, debido a que no están dadas las condiciones para su integración en la sociedad (Centro de Documentación y Estudios \& Bareiro, 2005).

\footnotetext{
* Cátedra de Salud Pública. Facultad de Odontología. Universidad del Pacífico Privada, Asunción, Paraguay.

"Estudiante del 5to año. Facultad de Odontología. Universidad del Pacífico Privada, Asunción, Paraguay.

"*** Cátedra de Odontología Preventiva. Facultad de Odontología. Universidad del Pacífico Privada, Asunción,Paraguay.
} 
PÉREZ BEJARANO, N. M.; ALARCÓN GONZÁLEZ, V. S.; FERREIRA GAONA, M. I.; DÍAZ REISSNER, C. V.; DURÉ, P.; ANDRIOTTI, N.; MORENO, D.; GALEANO, L.; MORA, A.; INSFRÁN, L. \& JARA, R. Estado de salud oral en discapacitados residentes en la Fundación Pequeño Cottolengo Don Orione, Paraguay. Int. J. Odontostomat., 10(1):69-74, 2016.

En tanto, en el consultorio odontológico se considera paciente con necesidades especiales a aquellos que presentan signos y síntomas de orden físico, mental o sensorial, social y/o de comportamiento anormales, que para su atención odontológica requiriere un tratamiento no rutinario (Tan Castañeda \& Rodríguez Calzadilla, 2001). La falta de conocimiento para desarrollar en ellos un tratamiento conservador, por parte del odontólogo llevan a las extracciones múltiples, sin tomar en cuenta las mutilaciones que ello significa, o bien directamente, el rechazo del paciente y la negatividad a su atención (Pirela de Manzano et al., 1999).

Es por esto que, para que la respuesta sea eficaz y eficiente se debe crear un cambio en la atención odontológica de pacientes especiales, basadas en estudios epidemiológicos que permitan determinar la magnitud y distribución del problema (Escudero, 2010; Malagon Valdez, 2007; Sáez Cuesta et al., 2008), teniendo en cuenta la intervención de factores externos, cuyo control contribuya a modificar la situación de salud oral (Escudero; Malagon Valdez).

Entre las discapacidades, una de las más frecuentes es el retraso mental, dada por causas que pueden ser genéticas, biológicas, psicosociales o socioculturales, consistente en un trastorno caracterizado por presentar una función intelectual inferior al de la media poblacional, con déficit o anomalías en la capacidad de aprendizaje y adaptación social (Dávila et al., 2005). La primera causa genética de retardo mental a nivel mundial es el Síndrome de Down (SD), cuyas manifestaciones bucales incluyen: lengua fisurada, macroglosia, protusión de la lengua, úvula bífida, erupción atrasada de los dientes primarios y permanentes, anormalidades de los dientes, en la corona y raíz, hipocalcificación del esmalte, prognatismo relativo y respiración oral, desarmonía oclusal; lo que puede constituir un factor de riesgo para desarrollar problemas de salud oral (Anselmi de Garriga \& Garriga García, 2002; Moret, 2004).

Otra discapacidad es el Trastorno del Espectro Autista (TEA), cuyo origen es multicausal, está caracterizada por presentar diversos cuadros clínicos acompañados de alteraciones del desarrollo con deficiencias en las áreas de comunicación (Irarrázaval et al., 2005; Maestre Vargas, 2006).

La Parálisis Cerebral (PC) es una patología que afecta a 2 de cada 1000 niños nacidos vivos a nivel mundial. Se considera la causa más frecuente de discapacidad física en niños después de haberse instaurado la vacuna de la poliomielitis. Es una disfunción motora debido a una lesión encefálica no progresiva que incide tempranamente en un sistema nervioso central inmaduro, el paciente presenta retraso en el desarrollo psicomotor, retardo mental, retardo en el lenguaje, epilepsia y trastornos sensoriales, pueden verse defectos de visión, audición, anormalidades en el habla y el lenguaje o alteraciones en la percepción; puede ir acompañada de retraso mental o de epilepsia (Malagon Valdez; Morales Chávez, 2008; Sáez Cuesta et al.). Se han encontrado una gran incidencia de anomalías dentomaxilares y disfunción oral en niños con parálisis cerebral, tales como: mordidas abiertas esqueletales, crecimiento dolicofacial, mordidas clase Il esqueletal, disfunción articulación témpora mandibular, asimetrías faciales e incompetencia labial. También pueden presentarse traumatismos dentales, bruxismo y hábitos como la interposición lingual, respiración bucal o deglución atípica. La incidencia de caries es similar a la población general pero las lesiones son más extensas debido a la deficitaria atención odontológica que reciben (Barrionuevo \& Solís, 2008; Navarro Melendro \& \& Restrepo Ibiza, 2005; Sáez Cuesta et al.; Soto-Veliz et al., 2009).

Se debe considerar que existen personas con discapacidad que no pueden recibir atención diaria por parte de sus familiares y que no cuentan con contención permanente. Motivo por el cual desde el año 1988 se creó el Pequeño Cottolengo, ubicado en el municipio de Mariano Roque Alonso en el Paraguay, con el fin de colaborar con la sociedad. Consistente en una obra de caridad destinada al cuidado de personas con alguna discapacidad (física, mental o ambas) abandonadas o que no cuentan con familiares ni recursos económicos para subsistir. Es una Asociación Civil sin fines de lucro que brinda a las personas servicios de por vida, brindándoles hogar, alimentos, vestimenta, medicación, rehabilitación, recreación y educación (http://discapacidadpcottolengo.blogspot.com).

En la actualidad en el ámbito odontológico existen métodos y técnicas que favorecen la atención de personas con discapacidad, es por ello, que como iniciativa este estudio pretende dar a conocer el estado bucal en el que se encuentran, y así incentivar a los profesionales a que hagan a un lado el temor de especializarse en el área ya que con una formación específica que les proporcione conocimientos y habilidades es fundamental para el cuidado y manejo de los mismos y de esta manera puedan mejorar su calidad de vida que les corresponde. 
PÉREZ BEJARANO, N. M.; ALARCÓN GONZÁLEZ, V. S.; FERREIRA GAONA, M. I.; DÍAZ REISSNER, C. V.; DURÉ, P.; ANDRIOTTI, N.; MORENO, D.; GALEANO, L.; MORA, A.; INSFRÁN, L. \& JARA, R. Estado de salud oral en discapacitados residentes en la Fundación Pequeño Cottolengo Don Orione, Paraguay. Int. J. Odontostomat., 10(1):69-74, 2016.

El objetivo fue describir el estado de salud oral de personas con discapacidades que viven en la Fundación Pequeño Cottolengo don Orione de Mariano Roque Alonso en Paraguay.

\section{MATERIAL Y MÉTODO}

El estudio fue observacional descriptivo de corte transversal. La población estuvo conformada por sujetos de ambos sexos con discapacidades de la Fundación Pequeño Cottolengo Don Orione en la ciudad de Mariano Roque Alonso en el año 2012. Fueron excluidos aquellos cuya situación de salud particular limiten su participación en el estudio.

Se realizó una prueba piloto en el Centro de Educación Especial Nuestra Señora del Rosario de la ciudad de Villeta como referencia para el cálculo del tamaño de la muestra, donde se obtuvo una prevalencia de caries del 99,0\%. Por tanto, para conseguir una precisión del $3 \%$ en la estimación de la proporción mediante un intervalo asintótico normal con corrección para poblaciones finitas al 99,0 \% bilateral, asumiendo que la prevalencia esperada de caries es de $100 \%$ y que el tamaño total de la población era de 63 , fue necesario incluir 35 sujetos.

Se solicitó permiso a las autoridades correspondientes y se trabajó con la colaboración de los odontólogos que asisten a los residentes del hogar. Además, se contó con el listado de residentes en el hogar, fueron seleccionados para formar parte del estudio mediante un muestreo no probabilístico de casos consecutivos, evaluados en tres visitas por dos odontólogas especialistas en odontopediatría, siguiendo las normas técnicas de la OMS para el examen oral (Organización Mundial de la Salud, 1997).

Se consideraron para el estudio las siguientes variables de interés: Demográficas (edad y sexo), presencia de caries (Índice de CPO-D), presencia de lesiones no cariosas, presencia de fluorosis (Índice de Dean), presencia de maloclusiones, presencia de fracturas (Índice de Basrani), presencia de lesiones que afectan a los tejidos blandos (Úlceras y tumoraciones) y presencia de factores de riesgo i) placa microbiana (Índice de Loë y Silness), ii) Dieta (Momentos de azúcar), iii) Saliva (Calidad) y iv) Higiene oral.

Los datos fueron tabulados en una planilla electrónica y analizados con el programa de Microsoft $\odot$
Excel 2013. Se aplicó estadística descriptiva donde se utilizaron distribución de frecuencia absoluta y relativa para las variables cualitativas, media y desvío estándar para las variables cuantitativas.

Se respetaron los principios universales de ética. El trabajo de investigación fue aprobado por el Comité de Ética de la Universidad del Pacífico Privada. Al finalizar el relevamiento se entregó un kit de higiene oral, que contenía un cepillo y pasta dental a todos los residentes del hogar. Los autores declaran no tener ningún conflicto de interés.

\section{RESULTADOS}

Al momento de recolección de datos la población total estaba conformada por 63 personas: entre ellos bebés, niños, jóvenes, adultos y ancianos, de ambos sexos. La muestra quedó conformada por 37 personas discapacitadas residentes en la fundación, que constituyen el $58,73 \%$ del total de residentes. La edad promedio fue de 32,51 (DE 14,58) años, con edades comprendidas entre 12 y 68 años, siendo el $54,04 \%$ hombres.

La condición de discapacidad predominante fue el retraso mental en el $89,89 \%$ de los casos, algunos presentaron de manera concomitante otras patologías como epilepsia, hipotiroidismo y parálisis cerebral. Además, se encontraron otras patologías como síndrome de Down en el 16,22 \%, siendo ésta la segunda condición más frecuente seguida de parálisis cerebral, autismo y paraplejia de miembros superiores e inferiores, con pocos casos.

El índice CPO-D fue en aumento conforme asciende el grupo etario, excepto el grupo de 41 a 50 años que disminuyó, encontrándose el índice más elevado en el grupo de 51 a 68 años. Se observó la misma tendencia para los componentes perdido $(P)$ y obturado $(\mathrm{O})$, sin embargo el componente cariado (C) disminuyó conforme ascendía el grupo etario (Fig. 1).

La patología más frecuente fue la maloclusión, hallada en el 86,49 \% de los casos, seguido de lesiones no cariosas y fracturas con $48,65 \%$ y $43,24 \%$ respectivamente, siendo la distribución por sexo similar en todas las patologías (Fig. 2). La fluorosis se presentó en el 2,70 \% de los sujetos.

En cuanto a los factores de riesgo, el $24,32 \%$ 
PÉREZ BEJARANO, N. M.; ALARCÓN GONZÁLEZ, V. S.; FERREIRA GAONA, M. I.; DÍAZ REISSNER, C. V.; DURÉ, P.; ANDRIOTTI, N.; MORENO, D.; GALEANO, L.; MORA, A.; INSFRÁN, L. \& JARA, R. Estado de salud oral en discapacitados residentes en la Fundación Pequeño Cottolengo Don Orione, Paraguay. Int. J. Odontostomat., 10(1):69-74, 2016.

tenía saliva viscosa; el indice de placa bacteriana fue de 2,04, mientras que, en ninguno de los casos los momentos de azúcar superaron tres, los cuidadores reportaron que a todos los sujetos una vez al día le realizan higiene oral.

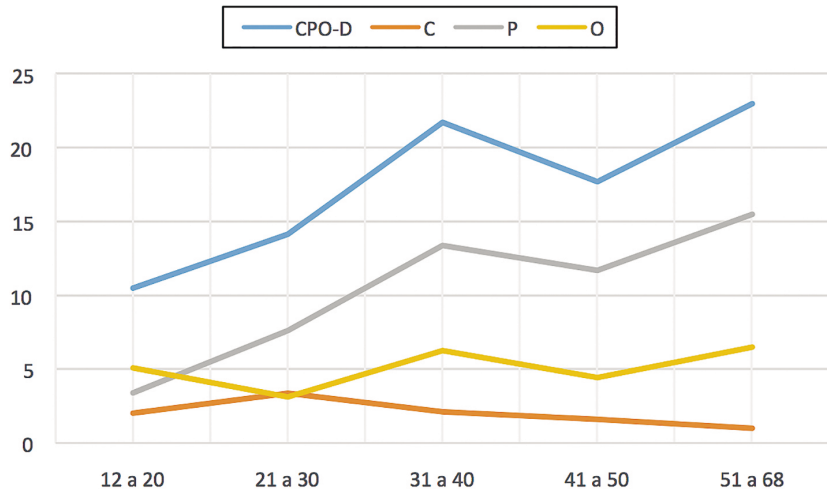

Fig. 1. Distribución del índice CPO-D y promedio de sus componentes por grupo etario. Fundación Pequeño Cottolengo Don Orione, Paraguay.

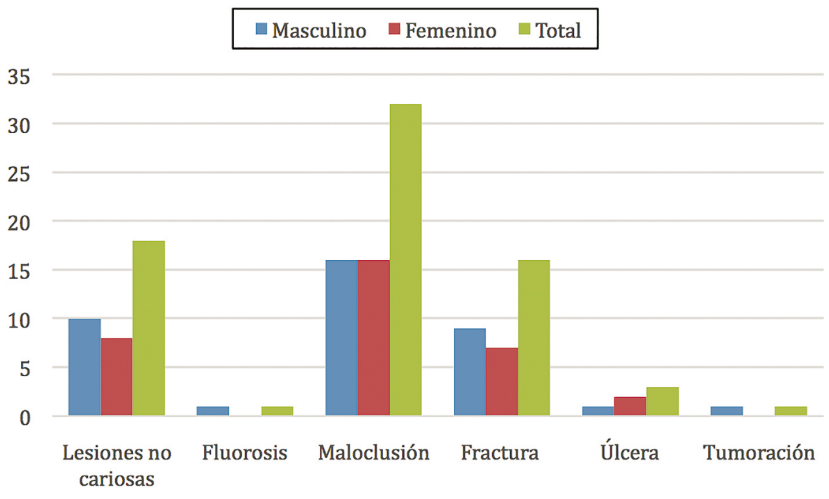

Fig. 2. Distribución de la presencia de patologías orales según sexo.Fundación Pequeño Cottolengo Don Orione, $\mathrm{Pa}$ raguay.

\section{DISCUSIÓN}

La OMS plantea que unos 600 millones de personas viven con discapacidades de diversos tipos. El $80 \%$ de ellas vive en países de bajos ingresos, la mayoría son pobres y no tienen acceso a servicios básicos ni a servicios de rehabilitación (Asamblea Mundial de la Salud). En la actualidad en Paraguay, según el censo 2002 del Instituto Nacional de Estadística y Censo estableció que, del total de la población paraguaya de 5163198 habitantes, aproximadamente un millón de personas presentaban alguna discapacidad y tan solo alrededor de cincuenta y cinco mil se encuentran registrados (Rivarola). Para la OMS, los estados deben asegurar la prestación de atención médica y servicios de rehabilitación a las personas con discapacidad, para que éstas logren alcanzar y mantener un nivel óptimo de autonomía y movilidad (Asamblea Mundial de la Salud).

Los datos de la OMS dicen que, las dos terceras partes de la población con discapacidades no reciben atención odontológica y, aunque los porcentajes estimados de población con discapacidad varían de una localidad a otra, los aspectos de salud integral para este grupo poblacional no incluyen su rehabilitación oral (Asamblea Mundial de la Salud).

En Paraguay están habilitados aproximadamente 16 centros de atención, educación y refugio a pacientes con discapacidades, entre éstos se encuentra la Fundación Pequeño Cottolengo con un total de 63 residentes, la muestra comprendida está formada por 20 hombres y 17 mujeres. El 57,14\% de las personas con discapacidad de la Fundación Pequeño Cottolengo presenta retardo mental, $11,11 \%$ retardo mental y enfermedades asociadas, $11,11 \%$ presentan síndrome de Down, con parálisis cerebral $9,5 \%$ y otras enfermedades el $11,11 \%$.

El índice CPO-D fue de 15,6 y la prevalencia de caries del $77 \%$ se obtuvo un índice CPO-D muy elevado comparado con Benavente Lipa (2007), que solo fue de 6,8 y 5,4 en pacientes con retardo mental.

Según los datos reportados por Morales Chavez, el $60 \%$ de la población presentaba maloclusión, y decía que su prevalencia es el doble en pacientes discapacitados que en la población general, coincidiendo con los resultados del estudio donde el $82 \%$ presenta maloclusión. También observó que el cepillado dental solo se realiza una vez al día, coincidiendo con este estudio.

Una investigación realizada por Serrano et al. (2012), señaló que el $82 \%$ de la población estudiada no presentaba fluorosis y un $8 \%$ la presentaban de forma muy leve, al igual que los resultados de este estudio en donde sólo el $5 \%$ la presenta.

El índice de placa bacteriana de Loë y Silness fue de 2,04; en comparación con en el estudio realizado en Colombia, donde dicho índice fue de 2,65 (Urbano et al., 2010), lo que refleja una deficiente higiene oral. En un estudio realizado a atletas con capacida- 
PÉREZ BEJARANO, N. M.; ALARCÓN GONZÁLEZ, V. S.; FERREIRA GAONA, M. I.; DÍAZ REISSNER, C. V.; DURÉ, P.; ANDRIOTTI, N.; MORENO, D.; GALEANO, L.; MORA, A.; INSFRÁN, L. \& JARA, R. Estado de salud oral en discapacitados residentes en la Fundación Pequeño Cottolengo Don Orione, Paraguay. Int. J. Odontostomat., 10(1):69-74, 2016.

des diferentes en Paraguay, un alto porcentaje acostumbra higienizarse los dientes una vez al día (Jacquett \& Chirife, 2012) de la misma manera que en este estudio en donde los encargados del Pequeño Cottolengo le realizan la higiene oral una vez al día.

Se concluye que, considerando la dificultad que conlleva inicialmente trabajar con pacientes con discapacidad, se ha obtenido una experiencia favorable, los residentes de la fundación han colaborado activamente desde su inicio, lo que permitió una mayor confianza por parte de ellos en el momento de realizar el levantamiento de datos. Es importante que busquemos iniciar un proceso donde se le otorgue más importancia en cuanto al cuidado de salud bucal para personas que se encuentren en esta condición, de modo que padres y encargados puedan brindar este cuidado mediante informaciones obtenidas y así poner en práctica el conocimiento adquirido en el mejoramiento de la salud oral de esta población.

PÉREZ BEJARANO, N. M.; ALARCÓN GONZÁLEZ, V. S.; FERREIRA GAONA, M. I.; DÍAZ REISSNER, C. V.; DURÉ, P.; ANDRIOTTI, N.; MORENO, D.; GALEANO, L.; MORA, A.; INSFRÁN, L. \& JARA, R. Oral health status of disabled residents at the Fundación Pequeño Cottolengo Don Orione, Paraguay. Int. J. Odontostomat., 10(1):69-74, 2016.

ABSTRACT: Approximately 600 million people worldwide live with disabilities, a figure that is increasing; $80 \%$ of these individuals live in low-income countries. In Paraguay, it is estimated that approximately $15 \%$ of the population has some type of disability. The objective of this study was to describe the state of oral health of people with disabilities who reside in the Fundación Pequeño Cottolengo Don Orione de Mariano Roque Alonso, Paraguay 2012. A descriptive crosssectional study was performed on 37 children, youth and adults aged between 12 and 68 years. We analyzed carious lesions thorough DMF-T index, lesions involving soft tissue and the presence of risk factors such as hygiene, microbial plaque, saliva and diet. The DMFT index was 16.24; presence of non-carious lesions was detected in $48.65 \%$ and $2.70 \%$ fluorosis. Level enamel fractures were found in $43.24 \%$ and oral hygiene was poor by Loë and Silness index. Of the population studied, $24.32 \%$ had viscous saliva and plaque index was 2.04 . While they did not exceed sugar times three in any cases; $100 \%$ of the population brushed once a day in the morning before breakfast. In the study population there are high values of missing and decayed teeth, therefore, awareness programs to promote the preservation of the teeth and oral health should be emphasized.

KEY WORDS: dental care for disabled, intellectual disability, group homes.

\section{REFERENCIAS BIBLIOGRAFICAS}

Anselmi de Garriga, A. L. \& Garriga García, E. Factores de riesgo, pesquisa y diagnóstico precoz en el cáncer de la cavidad bucal: Revisión de la literatura. Acta Odontol. Venez., 40(1):56-60, 2002.

Asamblea Mundial de la Salud, 58. Discapacidad, Incluidos la Prevención, el Tratamiento y la Rehabilitación: Informe de la Secretaría. Ginebra, OMS, 2005. Disponible en: http://apps.who.int//iris/handle/10665/22679

Barrionuevo, N. L. \& Solís, F. F. Anomalías dentó maxilares y factores asociados en niños con parálisis cerebral. Rev. Chil. Pediatr., 79(3):272-80, 2008.

Benavente Lipa, L. A. Influencia del nivel de educación sanitaria de los padres o apoderados en el estado de salud e higiene bucal del niño con retardo mental. Odontol. Sanmarquina, 10(2):3-7, 2007.

Centro de Documentación y Estudios (CDE) \& Bareiro, L. Discriminaciones. Debate Teórico Paraguayo / Legislación Antidiscriminatoria. Asunción, CDE, UNFPA, Comisión de Equidad, Género y Desarrollo Social de la Cá- mara de Senadores, la Comisión de Equidad Social y Género de la Cámara de Diputados, la Comisión de Derechos Humanos y Asuntos Indígenas de la Cámara de Diputados, 2005.

Escudero, J. C. Esbozo de la educación especial en Chile: 1850-1980. Rev. Educ. Pedagog., 22(57):31-49, 2010.

González, D. Discapacidad en los Censos de 2010. Los Censos de 2010 en América Latina: Balance y Principales Lecciones Aprendidas. Santiago de Chile, Centro Latinoamericano y Caribeño de Demografía CELADE-División de Población de la CEPAL, 2013. Recuperado a partir de http://www.cepal.org/celade/noticias/paginas/4/ 51424/Daniela_Gonzalez_Celade.pdf

Irarrázaval, O. M. E.; Brokering, A. W. \& Murillo, B. G. A. Autismo: una mirada desde la psiquiatría de adultos. Rev. Chil. Neuro-Psiquiatr., 43(1):51-60, 2005.

Jacquett, N. L. \& Chirife, M. T. Historia dental de atletas con capacidades diferentes de la ciudad de Asunción Paraguay. Parag. Oral Res., 2(1):5-10, 2012. 
PÉREZ BEJARANO, N. M.; ALARCÓN GONZÁLEZ, V. S.; FERREIRA GAONA, M. I.; DÍAZ REISSNER, C. V.; DURÉ, P.; ANDRIOTTI, N.; MORENO, D.; GALEANO, L.; MORA, A.; INSFRÁN, L. \& JARA, R. Estado de salud oral en discapacitados residentes en la Fundación Pequeño Cottolengo Don Orione, Paraguay. Int. J. Odontostomat., 10(1):69-74, 2016.

Maestre Vargas, N. La Homeopatía y el trastorno del espectro autista. Gac. Homeopat. Caracas, 14(1):17-24, 2006.

Malagon Valdez, J. Parálisis cerebral. Medicina (B. Aires), 67(6):586-92, 2007.

Morales Chávez, M. C. Patologías bucodentales y alteraciones asociadas prevalentes en una población de pacientes con parálisis cerebral infantil. Acta Odontol. Venez., 46(1):52-5, 2008.

Moret, Y. Enfermedades genéticas que afectan la cavidad bucal. Revisión de la literatura. Acta Odontol. Venez., 42(1):59-66, 2004.

Navarro Melendro, A. M. \& Restrepo Ibiza, A. P. Consecuencias neuropsicológicas de la parálisis cerebral estudio de caso. Univ. Psychol., 4(1):107-15, 2005.

Olmedo Zorilla, E. El 50\% de los discapacitados está así a causa de algún accidente de tránsito. Asunción, ABC Color, 2011. Disponible en: http://www.abc.com.py/ edicion-impresa/locales/el-50-de--los---discapacitadosesta-asi-a-causa-de-algun-accidente-de-transito303876.html

Organización Mundial de la Salud. Encuestas de Salud Bucodental: Métodos Básicos. 4a ed. Ginebra, Organización Mundial de la Salud, 1997.

Pirela de Manzano, M. A.; Salazar V, C. R. \& Manzano, F. M. A. Patología bucal prevalente en niños excepcionales. Acta Odontol. Venez., 37(3):193-8, 1999.

Rivarola, M. Para Dejar de Ser Invisibles. Asunción, ABC Color, 2012. Disponible en: http://www.abc.com.py/especiales/fin-de-semana/para-dejar-de-ser-invisibles447721.html

Sáez Cuesta, U.; Castejón Navas, I.; Molina Escribano, A. \& Roncero Goig, M. Estudio epidemiológico de Salud Bucodental en pacientes con Parálisis Cerebral. Rev. Clin. Med. Fam., 2(5):206-9, 2008.

Serrano, M.; Torrelles, A. \& Simancas, P- Y. C. Estado de salud bucodental en niños con discapacidad intelectual. Acta Odontol. Venez., 50(3), 2012. Disponible en: http:/ /www.actaodontologica.com/ediciones/2012/3/art5.asp

Soto-Veliz, R.; Vallejos-Ragas, R.; Campos-Sánchez, R.; Ouslanova, P.; Agüero Cortez, Z.; Bernal-Garayar, N.; Camargo Hurtado, J. \& De la Cruz Calvo, C. Situación bucal de pobladores con discapacidades en el distrito de San Luis-Lima. Rev. Estomatol. Herediana, 19(1):820, 2009.

Stang Alva, M. F. Las Personas con Discapacidad en América Latina: del Reconocimiento Jurídico a la Desigualdad Real. Vitacura, Comisión Económia para America
Latina (CEPAL), 2011. Disponible en: http:// www.cepal.org/es/publicaciones/7135-personasdiscapacidad-america-latina-reconocimiento-juridico-ladesigualdad-real

Tan Castañeda, N. \& Rodríguez Calzadilla, A. Correspondencia entre la formación académica del estomatólogo relacionado con pacientes especiales y la práctica estomatológica integral. Rev. Cuba. Estomatol., 38(3):181-91, 2001.

Urbano, D. L. C.; Cabrera, M. I. P. \& Caiza, G. A. A. Higiene oral de niños, adolescentes y jóvenes con discapacidad cognitiva leve. Univ. Salud, 1(12):34-42, 2010.

Vázquez Barrios A, \& Cáceres N. El Abordaje de la Discapacidad Desde la Atención Primaria de Salud. Buenos Aires, Organización Panamericana de la Salud (OPS), 2008. Disponible en: http://publicaciones. ops.org.ar/publicaciones/otras\%20pub/discapacidad.pdf

Dirección para Correspondencia:

Clarisse Díaz Reissner

Cátedra de Salud Pública

Facultad de Odontología

Universidad del Pacífico Privada

Asunción

PARAGUAY

Email: diazclarisse@gmail.com

Recibido: 16-07-2015

Aceptado: 01-04-2016 